Chirurg 2009 $\cdot 80: 85-87$

DOI 10.1007/s00104-008-1616-5

Online publiziert: 16.01.2009

๑) Springer Medizin Verlag 2009

H. Dralle

Universitätsklinik für Allgemein-, Viszeral- und Gefäßchirurgie, Universitätsklinikum Halle, Medizinische Fakultät der Martin-Luther Universität Halle-Wittenberg, Halle

\title{
Endokrine Chirurgie: Quo vadis?
}

Wo liegt die Zukunft der endokrinen Chirurgie? Kann eine Subspezialität wie die endokrine Chirurgie mit vier Organbereichen (Abdomen, Retroperitoneum, Thorax, Hals) in der Kompetition mit den Organspezialisten heute noch bestehen? Kann eine Subspezialität, die je nach klinikeigenem Schwerpunkt ein operatives Volumen von durchschnittlich nicht mehr als 5-10\% umfasst und bei der ca. 95\% der Eingriffe außerhalb des Fachmittelpunktes liegen, bei der Facharztanwärter in der Regel nur über Erfahrungen von ca. 1o Operationen beim häufigsten Eingriff (Schilddrüse), bei adrenalen und pankreatischen endokrinen Operationen jedoch über fast keine Erfahrungen verfügen $[2,3]$, konkurrenzfähig zu den Organspezialisten des eigenen Fachs oder anderer Fächer sein? Ist eine Subspezialität wie die endokrine Chirurgie auf einem guten Weg, wenn international gesehen seit 1015 Jahren nur noch ein verschwindend kleiner Anteil endokriner Chirurgen die endokrine Chirurgie als Ganzes in praxi beherrscht? Dieses Schwerpunktheft möchte anhand der Themen, aber auch ihrer Autoren beispielhaft aufzeigen, dass endokrine Chirurgie nicht nur ein außerordentlich spannendes Gebiet mit hohem translationalem Innovationspotenzial ist, sondern auch ein Gebiet, dass als Gesamtgebiet mit all seinen Facetten durchaus beherrschbar ist, wenn es in die profunden Kenntnisse und Erfahrungen der Viszeralchirurgie eingebettet bleibt.

Die endokrine Chirurgie als Subspezialität der Allgemein-/Viszeralchirurgie hat ihre entscheidenden Impulse Anfang der 5oer Jahre des vergangenen Jahrhunderts vor allem aus England, USA und Skandinavien erhalten, nachdem sich die Endo- krinologie als wissenschaftliches Gebiet zu etablieren begann, vor allem nachdem 1948 Kortison in die Therapie eingeführt wurde und Adrenalektomien und Hypophysektomien erstmals sicher durchgeführt werden konnten [10]. Zahlreiche neue Krankheitsbilder und deren chirurgische Therapiemöglichkeiten wurden in der Folgezeit erkannt (z. B. Conn-Syndrom, Zollinger-Ellison-Syndrom, medulläres Schilddrüsenkarzinom, multiple endokrine Neoplasie).

\section{> Endokrine Chirurgie - ein spannendes Gebiet mit hohem translationalem Innovationspotenzial}

In den Jahren von 1950 bis 1980 erlebte die endokrine Chirurgie durch herausragende, zumeist aus der Allgemeinchirurgie kommende Persönlichkeiten einen ungeheuren Aufschwung wie z. B. Oliver Cope (Boston), Oliver Beahrs (Mayo Clinic Rochester), George Gile Jr. (Cleveland Ohio), Selwyn Taylor und Richard Welbourne (Hammersmith London), James Priestley (Mayo Clinic Rochester), Stanley Friesen (Kansas City), Ivan Johnston (Newcastle-upon-Tyne) und Per-Ola Granberg (Karolinska Stockholm). Ende der 7oer Jahre formierten sich endokrin-chirurgische Gesellschaften in Skandinavien, England, Japan, Fankreich und Italien. 1979 wurde auf Initiative von Peter Heimann (Bergen, Norwegen) die International Association of Endocrine Surgeons (IAES), 1980 die American Association of Endocrine Surgerons (AAES) und 1982 auf Initiative von Hans Röher die Chirurgische Arbeitsgemeinschaft Endo- 
krinologie (CAEK) der Deutschen Gesellschaft für Chirurgie gegründet [6].

Seit den goer Jahren haben jedoch Entwicklungen in der endokrinen Chirurgie eingesetzt, die trotz zunehmender Sicherheit bei Primär- und Rezidiveingriffen [7], trotz großer Fortschritte bei der paradigmatischen Einführung und Etablierung DNA-basierter prophylaktischer Operationen wie z. B. beim medullären Schilddrüsenkarzinom $[1,4,11]$ zu einer zunehmenden Dissoziation und Separierung von zervikaler und abdominell/retroperitonealer endokriner Chirurgie geführt haben. Auch wenn die wissenschaftliche Einheit der endokrinen Chirurgie durch die publikatorische Aktivität großer internationaler endokrin-chirurgischer Zentren bislang nicht gefährdet erscheint, wie Lehrbücher, nationale und internationale Kongresse zeigen, hat sich die klinische Realität davon jedoch weit entfernt. International wird heute endokrine Zervikalchirurgie zunehmend, bisweilen ausschließlich, durch Hals-Nasen-Ohren-Chirurgen, thorakale endokrine Chirurgie durch Thoraxchirurgen, Nebennierenchirurgie durch Urologen und endokrine Gastrointestinalchirurgie durch spezialisierte Digestivtraktchirurgen durchgeführt.

Auf nationaler und internationaler Ebene wurde dieser Entwicklung durch eine Reihe bedeutsamer Entscheidungen Rechnung getragen. Jüngste Beispiele sind die 2007 vollzogene Öffnung der American Association of Endocrine Surgeons für HNO-Kollegen und Kollegen anderer chirurgischer Fächer mit endokrinem Zielorgan $[5,8]$ und die jetzt erfolgte Umbenennung der British Association of Endocrine Surgeons (BAES) in British Association of Endocrine and Thyroid Surgeons (BAETS) unter gleichzeitigem Einschluss von HNO-Chirurgen. Im europäischen Raum gibt es darüber hinaus auf der Ebene der chirurgischen Fachzuordnung und Weiterbildung einige bemerkenswerte Beispiele für Schritte in die genannte Richtung: In Dänemark wurde jetzt offiziell die Schilddrüsenchirurgie der HNO zugeordnet; bei der UEMS wird seit 2008 durch die Division of Endocrine Surgery nach bestandener Prüfung nicht nur das Zertifikat „Endokrine Chirurgie“, sondern auch „Schilddrüsen- und Nebenschilddrüsenchirurgie" vergeben; in Norwegen wurde die endokrine und Mammachirurgie als eigenständige chirurgische Subdisziplin begründet und es wird überlegt, ein entsprechendes Kurrikulum direkt aufbauend auf einer kurzen Basischirurgie zu entwickeln. Alle diese Beispiele zeigen, dass unübersehbar ein derzeit wachsender Trend von der systemorientierten zur organ- bzw. regionspezifischen Chirurgie stattfindet.

Quo vadis endokrine Chirurgie? Am meisten in Gefahr, der Allgemein-/Viszeralchirurgie wie auch schon andere Gebiete (z. B. Mammachirurgie) verloren zu gehen, ist aufgrund der Dislokation und Besonderheiten des Halsgebietes im Vergleich zum Abdominal- und Retroperitonealraum die endokrine Zervikalchirurgie. Die thorakale endokrine Chirurgie wird auch in Deutschland ohnehin schon fast ausschließlich von spezialisierten Thoraxchirurgen durchgeführt. Die Chirurgie von Schilddrüse und Nebenschilddrüsen hat sich aufgrund der zahlenmäßigen Dominanz innerhalb der endokrinen Chirurgie (ca. $95 \%$ ) zu einem Gebiet entwickelt, das unter verschiedenen Aspekten beträchtliche Attraktivität besitzt (Standardisierung, organbezogene Techniken und Ausbildung, Pathophysiologie, nahezu ausschließlich elektive Chirurgie) und wegen der anatomischen Nähe und der infolge neoadjuvanter Therapieverfahren erheblich reduzierten Operationen plattenepithelialer Kopf-Hals-Tumoren kompensatorisch ein von der HNO-Chirurgie bevorzugtes neues Operationsgebiet darstellt. Die Gefahr, dass auch in Deutschland, wo die endokrine Chirurgie noch fester Bestandteil der Viszeralchirurgie ist, insbesondere die zervikale und retroperitoneale endokrine Chirurgie der Viszeralchirurgie verloren geht, kann abgesehen von entsprechenden DRG/Budgetinitiativen nur durch Kompetenz in chirurgischer Technik, Kenntnis der Pathophysiologie, Ausund Weiterbildung, Forschung und Entwicklung gebannt werden.

Da die endokrine Chirurgie als Ganzes nur innerhalb der Viszeralchirurgie als systembezogene Einheit lebensfähig ist, wäre die Loslösung von der Viszeralchirurgie fatal. Dies erfordert allerdings die Bewältigung mehrerer Herausforderungen:

- die kompetente Kompetition und Kooperation mit den auf den jeweiligen anatomischen Regionen tätigen operativen Fachgebieten,

- die aktive Weiterentwicklung der viszeralen endokrinen Chirurgie durch Öffnung zu neuen Techniken wie z. B. minimal-invasiven Verfahren, organübergreifenden Resektions- und Rekonstruktionsverfahren im Halsbereich, funktionserhaltender Chirurgie bei Nebennieren- und neuroendokrinen Pankreastumoren, intraoperativem Neuromonitoring und intraoperativer Hormonbestimmungen (s. Beiträge in diesem Heft),

- die Akzeptanz und Schaffung entsprechender Weiterbildungsstrukturen,

- die Etablierung evidenzbasierter Qualitätskriterien, die Ermöglichung universitärer Forschung in der endokrinen Chirurgie und vor allem

- die Eröffnung von Berufsperspektiven in Schwerpunktkrankenhäusern und Universitäten für Nachwuchs-Viszeralchirurgen mit endokrinem Schwerpunkt.

Leider haben bereits mehrere jüngere endokrine Chirurgen aus Mangel an entsprechenden Berufsperspektiven Deutschland verlassen.

Die endokrine Chirurgie als Schwerpunkt innerhalb der Allgemein-/Viszeralchirurgie ist in praxi in vielen Ländern schon nicht mehr Realität, aber auch in Deutschland gerade wegen dieser globalen Entwicklungen in Gefahr.

Die in diesem Heft von kompetenten Viszeralchirurgen verfassten Beiträge zeigen das aktuelle Spektrum und neue Entwicklungen im Bereich der endokrinen Chirurgie, Techniken und Konzepte, die aus der Viszeralchirurgie entwickelt wurden und die Vitalität dieses spannenden Gebietes belegen. In weiser Einschätzung der gegenwärtigen und zukünftigen Chancen der endokrinen Chirurgie hat Norman W. Thompson [9] schon vor über 10 Jahren erkannt, dass „Enhancement" anstelle von „Fragmentation“ die wesentliche Voraussetzung für den Erhalt der endokrinen Chirurgie als ganzheitliches, systemorientiertes Konzept innerhalb der Vis- 
zeralchirurgie darstellt. Beides, d. h. Weiterentwicklung und Vermeidung weiterer Aufsplitterung mit zwangsläufig resultierendem Verlust an andere Fachgebiete, ist allerdings nur dann möglich, wenn die endokrine Chirurgie aufbauend auf der Viszeralchirurgie in ihr als Subspezialität eingebettet bleibt und Nachwuchschirurginnen und -chirurgen des Gebietes angemessene eigenständige Berufsperspektiven eröffnet werden.
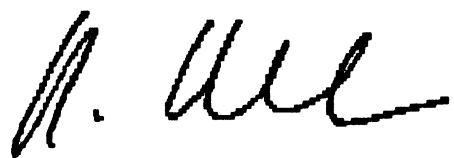

H. Dralle

\section{Korrespondenzadresse}

\section{Prof. Dr. H. Dralle FRCS, FACS}

Universitätsklinik für Allgemein-, Viszeralund Gefäßchirurgie, Universitätsklinikum Halle, Medizinische Fakultät der MartinLuther Universität Halle-Wittenberg Ernst-Grube-Straße 40, 06097 Halle henning.dralle@medizin.uni-halle.de

\section{Literatur}

1. Dralle H, Gimm O, Simon D et al (1998) Prophylactic thyroidectomy in 75 children and adolescents with hereditary medullary thyroid carcinoma: german and austrian experience. World J Surg 22:744-751

2. Harness JK, Organ CH, Thompson NW (2009) Operative experience of $U \mathrm{~S}$ general surgery residents with disease of the adrenal glands, endocrine pancreas and other less common endocrine organs (in press)

3. Le D, Karmali S, Harness JK et al (2008) An update: The operative experience in adrenal, pancreatic and other less common endocrine disease of $U$ S general surgery residents. World J Surg 32:232236

4. Lips CJM, Landsvater RM, Höppener JWM et al (1994) Clinical screening as compared with DNA analysis in families with multiple endocrine neoplasia type 2a. N Engl J Med 331:828-835

5. Randolph GW, Healy GB (2008) Otolaryngology and the american association of endocrine surgery: time for a change. Surgery 143:153-154

6. Röher HD, Wahl RA (1983) Chirurgische Endokrinologie Thieme, Stuttgart

7. Rothmund $M$ (2005) Wiederholungseingriffe in der endokrinen Chirurgie. Chirurg 76:205-206

8. Thompson GB (2007) Editorial: The maturation of a specialty: workforce projections for endocrine surgery. Surgery142:884-886

9. Thompson NW (1996) The evolution of endocrine surgery as a subspecialty of general surgery. Arch Surg 131:456-471

10. Welbourne RB (1990) The history of endocrine surgery. Praeger, New York

\section{Liebe Leserinnen, liebe Leser,}

„Der Chirurg“ bietet Ihnen jeden Monat umfassende und aktuelle Beiträge zu interessanten Themenschwerpunkten aus allen Gebieten der operativen Medizin.

Möchten Sie ein bereits erschienenes Heft nachbestellen? Die folgenden Ausgaben können Sie direkt bei unserem Kundenservice zum Preis von je EUR 32,- beziehen:

\section{8}

- 1/2008 Chirurgie des Mediastinums

- 2/2008 Lebertransplantation

- 3/2008 Allgemeinchirurgie in der Diskussion

- 4/2008 Peritonitis

- 5/2008 Aktuelle Proktologie

- 6/2008 Die chronische Wunde

- 7/2008 Gastrointestinale Stromatumoren

- 8/2008 Benigne Lebertumoren inkl. Echinococcus

- 9/2008 Morbide Adipositas

- 10/2008 Wirbelsäulenchirurgie

- 11/2008 Hereditäre Karzinome

- 12/2008 Aktuelle Techniken in der Pankreaschirurgie - Teil 1

\section{9}

- 1/2009 Aktuelle Techniken in der Pankreaschirurgie - Teil 2

- 2/2009 Neue Konzepte in der Endokrinen Viszeralchirurgie

- 3/2009 Weichteilsarkome

- 4/2009 Rektumkarzinom

- 5/2009 Diabetes mellitus

- 6/2009 Thrombozytenaggregationshemmung
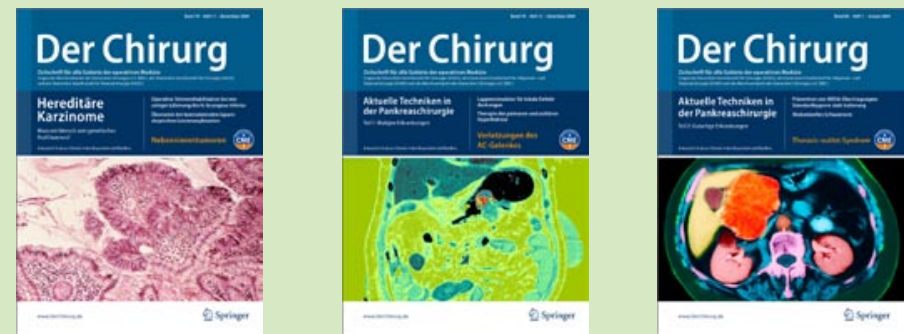

\section{So erreichen Sie unseren}

\section{Kundenservice:}

Springer Customer Service Center GmbH

Haberstr. 7

69126 Heidelberg

Tel.: +496221345-4303

Fax: +49 $6221345-4229$

E-Mail: Leserservice@springer.com

\section{www.DerChirurg.de}

